$\begin{aligned} & \text { No frontiers } \\ & \text { Europe and China } \\ & \text { embark on cosmic } \\ & \text { collaboration } \\ & \text { p106 }\end{aligned}$
$\ldots \ldots \ldots$

\title{
Genetics group targets disease markers in the human sequence
}

\section{David Adam, London}

Researchers are planning a new publicprivate consortium to map genetic variation across the entire human genome.

The proposed collaboration between several biotechnology companies and public laboratories, including the Sanger Centre in Britain and the Whitehead Institute for Biomedical Research in Cambridge, Massachusetts, aims to produce a publicly available genetic map of some 300,000 'haplotypes' within two years.

Haplotypes are sets of genetic markers that are close enough on a chromosome to be inherited together. They can be used to unravel the genetic differences that make some people more susceptible than others to conditions such as diabetes and heart disease.

Plans for the \$60-million project will be discussed at a meeting in Washington on 18-19 July. The US National Institutes of Health and the Wellcome Trust, the UKbased charity that funds the Sanger Centre, are keen to support the consortium, and several pharmaceutical companies have also been approached for possible funds.

"The objective is to create a framework map across the entire genome that will be of great use in disease-gene finding, in pharmacogenetics and in evolutionary studies," says Lon Cardon, head of bioinformatics at the Wellcome Trust Centre for Human Genetics in Oxford.

Cardon says the proposed consortium would build on both the human genome sequencing project and the recently completed effort to identify single-nucleotide polymorphisms (SNPs). The latter collaboration identified some two million SNPs sites where the genetic code between individuals differs by just one base.

"Now we have the potential to actually do something with those variations by seeing how they are associated across the genome," Cardon says.

SNPs act as markers for genetics researchers sifting the genome for diseasecausing genes. They allow genes to be traced through generations, for example, and can flag up genetic differences between people affected and unaffected by a disease.
But using SNPs alone can be difficult and expensive, partly because it is hard to trace individual SNPs in a genome containing three billion base pairs. Using haplotypes eases some of these difficulties, as each contains a group of SNPs that tend to be inherited together. So the two million or so separated markers are consolidated into more manageable clusters, making it easier to identify variation in the genome.

Haplotypes are found by analysing genotype data. The new consortium would essentially be a high-throughput genotyping effort, in much the same way that the public human genome project is a highthroughput sequencing effort.

The consortium would produce a reference map of haplotype regions across the genome. Researchers studying diabetes, for example, would then be able to see which haplotype regions differ in affected individuals, helping to narrow down the region of the genome associated with the condition.

"It's more informative if you can link two markers together in the inheritance pattern than just use one marker or the other," says Mike Boyce-Jacino of Orchid BioSciences, a biotechnology firm based in Princeton, New

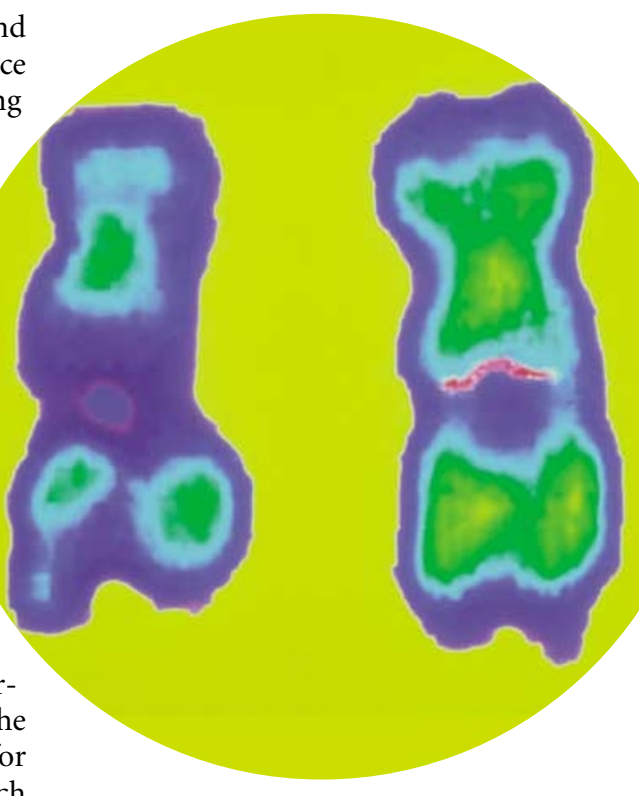

Missing link: the chromosome on the left lacks a gene (red) that may protect against cancer.

Jersey, and one of the companies involved. "What you're really trying to do is to reduce the background noise when you go in to look for a genetic association with the inheritance of a disease," he says.

\section{Physicists show what really matters}

\section{Colin Macilwain, Washington}

Researchers at the Stanford Linear

Accelerator Center (SLAC) in California

have confirmed an important tenet of the standard model of high-energy physics. They have successfully measured a parameter that may help to explain the preponderance of matter over antimatter in the Universe.

An international team of 600 physicists announced on 6 July that they had measured the parameter, which expresses the degree of asymmetry between heavy subatomic particles called B mesons and their corresponding anti-B mesons.

The long-awaited finding, based on the observation of 32 million decay events by a 1,200-tonne detector called BaBar, confirms the existence of the overall asymmetry, known as charge-parity $(\mathrm{CP})$ violation.

"We're absolutely delighted," says Stewart Smith, a physicist at Princeton University and spokesman for the experiment. "We expected it would take another year to get this far."

The 'B Factory' experiment, which generated the result, was devised and built by a team led by Jonathan Dorfan, who is now director of SLAC (see Nature 403, 586; 2000).

A rival international team at the KEK particle physics laboratory in Japan is set to announce its own measurement of $\mathrm{CP}$ violation at a news conference next week. 